\title{
Existence and uniqueness of solutions for a class of fractional nonlinear boundary value problems under mild assumptions
}

\author{
Imed Bachar ${ }^{1 *}$ (D), Habib Mâagli ${ }^{2,3}$ and Hassan Eltayeb
}

"Correspondence:

abachar@ksu.edu.sa

${ }^{1}$ Mathematics Department, College

of Science, King Saud University, P.O.

Box 2455, 11451, Riyadh, Saudi

Arabia

Full list of author information is

available at the end of the article

\section{Abstract}

We deal with the following Riemann-Liouville fractional nonlinear boundary value problem:

$$
\left\{\begin{array}{l}
\mathcal{D}^{\alpha} v(x)+f(x, v(x))=0, \quad 2<\alpha \leq 3, x \in(0,1), \\
v(0)=v^{\prime}(0)=v(1)=0 .
\end{array}\right.
$$

Under mild assumptions, we prove the existence of a unique continuous solution $v$ to this problem satisfying

$$
|v(x)| \leq c x^{\alpha-1}(1-x) \quad \text { for all } x \in[0,1] \text { and some } c>0 .
$$

Our results improve those obtained by Zou and He (Appl. Math. Lett. 74:68-73, 2017).

MSC: 34A08; 34B15; 34B27

Keywords: Fractional differential equation; Green's function; Existence and uniqueness of solution; Banach's contraction principle

\section{Springer}

\section{Introduction}

Fractional differential equations have attracted great attention due to their ability to model various phenomena in applied sciences. The so-called fractional differential equations are specified by generalizing the standard integer-order derivative to arbitrary order. For more interesting theoretical results and scientific applications of fractional differential equations, we refer to the monographs of Diethelm [2] and Kilbas et al. [3] and references therein.

The existence, uniqueness, and global behavior of solutions for boundary value problems of fractional differential equations have been considered in several recent papers (see, e.g., $[1,4-9]$ and references therein).

(c) The Author(s) 2021. This article is licensed under a Creative Commons Attribution 4.0 International License, which permits use, sharing, adaptation, distribution and reproduction in any medium or format, as long as you give appropriate credit to the original author(s) and the source, provide a link to the Creative Commons licence, and indicate if changes were made. The images or other third party material in this article are included in the article's Creative Commons licence, unless indicated otherwise in a credit line to the material. If material is not included in the article's Creative Commons licence and your intended use is not permitted by statutory regulation or exceeds the permitted use, you will need to obtain permission directly from the copyright holder. To view a copy of this licence, visit http://creativecommons.org/licenses/by/4.0/. 
Zou and He [1] investigated the problem

$$
\left\{\begin{array}{l}
\mathcal{D}^{\alpha} v(x)+f(x, v(x))=0, \quad 2<\alpha \leq 3, x \in(0,1), \\
v(0)=v^{\prime}(0)=v(1)=0
\end{array}\right.
$$

where $\mathcal{D}^{\alpha}$ denotes the standard Riemann-Liouville fractional derivative, and $f$ satisfies the following conditions:

(H1) $f \in C((0,1) \times \mathbb{R}, \mathbb{R})$ and $\int_{0}^{1}|f(x, 0)| d x<\infty$;

(H2) There exists $q \in C((0,1),[0, \infty))$ such that

$$
|f(x, v)-f(x, w)| \leq q(x)|v-w|, \quad \forall x \in(0,1), v, w \in \mathbb{R},
$$

and

$$
0<\int_{0}^{1} q(x) d x<\infty
$$

Let $L>0$ be the minimum positive constant such that

$$
\int_{0}^{1} G_{\alpha}(x, y) q(y) y^{\alpha-1}(1-y) d y \leq L x^{\alpha-1}(1-x),
$$

where $G_{\alpha}(x, y)$ is the Green's function (given later in this paper) associated with problem (1.1). By using Banach's contraction principle on some convenient Banach space they have obtained the following result.

Theorem 1.1 Under assumptions $(\mathrm{H} 1)-(\mathrm{H} 2)$ and $L<1$, problem (1.1) has a unique solution in $C([0,1])$.

Motivated by this reault, we prove that the conclusion of Theorem 1.1 remains true under the following weaker assumptions:

(A1) $f \in C((0,1) \times \mathbb{R}, \mathbb{R})$ and $\int_{0}^{1}(1-x)^{\alpha-2}|f(x, 0)| d x<\infty$;

(A2) There exists $q \in C((0,1),[0, \infty))$ such that

$$
|f(x, v)-f(x, w)| \leq q(x)|v-w|, \quad \forall x \in(0,1), v, w \in \mathbb{R},
$$

and

$$
0<M_{q, \alpha}:=\frac{1}{\Gamma(\alpha-1)} \int_{0}^{1} x^{\alpha-1}(1-x)^{\alpha-1} q(x) d x<\infty .
$$

Remark 1.2 It is clear that conditions (H1)-(H2) imply (A1)-(A2).

Conversely, for $\beta \in[1, \alpha-1)$, the function $f(x, v):=(1-x)^{-\beta}(1+v)$ satisfies hypotheses (A1)-(A2) but not conditions (H1)-(H2). So assumptions (A1)-(A2) are weaker.

In this paper, for $\alpha \in[2,3)$, we use the following notations:

- $h(x):=x^{\alpha-1}(1-x), x \in[0,1]$.

- $G_{\alpha}(x, y)$ denotes the Green's function of the operator $v \rightarrow-D^{\alpha} v$ with boundary conditions $v(0)=v^{\prime}(0)=v(1)$. 
- $E:=\left\{a>0: \int_{0}^{1} G_{\alpha}(x, y) h(y) q(y) d y \leq a h(x), x \in[0,1]\right\}$ (we will see that $E \neq \emptyset$ ).

$\bullet M:=\inf E$.

We will prove that $M$ is a positive constant satisfying the following range estimation:

$$
M_{q, \alpha+1} \leq M \leq M_{q, \alpha}
$$

- For $a \in \mathbb{R}, a^{+}:=\max (a, 0)$.

- $C_{h}([0,1]):=\{v \in C([0,1])$ : there is $\sigma>0$ such that $|v(x)| \leq \sigma h(x), x \in[0,1]\}$.

In the next remark, we list some properties of elements of $C_{h}([0,1])$.

\section{Remark 1.3}

(i) $C_{h}([0,1])$ is a Banach space equipped with the following $h$-norm:

$$
\|v\|_{h}:=\inf \{\sigma>0:|v(x)| \leq \sigma h(x), x \in[0,1]\}=\sup _{x \in(0,1)} \frac{|v(x)|}{h(x)} .
$$

(ii) $v \in C_{h}([0,1])$ if and only if $v=h \varphi$, where $\varphi$ is a bounded continuous function in $(0,1)$.

Our main result is the following:

Theorem 1.4 Assume that (A1) and (A2) hold. If $M<1$, then problem (1.1) has a unique solution $v$ in $C_{h}([0,1])$. In addition, for any $v_{0} \in C_{h}([0,1])$, the iterative sequence $v_{k}(x):=$ $\int_{0}^{1} G_{\alpha}(x, y) f\left(y, v_{k-1}(y)\right) d y$ converges to $v$ with respect to the h-norm, and we have

$$
\left\|v_{k}-v\right\|_{h} \leq \frac{M^{k}}{1-M}\left\|v_{1}-v_{0}\right\|_{h}
$$

Our paper is organized as follows. In Sect. 2, we improve the estimates on Green's function $G_{\alpha}$ obtained in [1, Lemma 2.2]. This allows us to obtain the range estimation (1.6). Our main result is proved in Sect. 3. Some examples and approximations are given at the end.

\section{Preliminaries}

Definition 2.1 ([3]) Let $f:(0, \infty) \rightarrow \mathbb{R}$ be a measurable function.

(i) The Riemann-Liouville fractional integral of order $\gamma>0$ for $f$ is defined as

$$
I^{\gamma} f(x):=\frac{1}{\Gamma(\gamma)} \int_{0}^{x}(x-y)^{\gamma-1} f(y) d y
$$

where $\Gamma$ is the Euler gamma function.

(ii) The Riemann-Liouville fractional derivative of order $\gamma>0$ for $f$ is defined as

$$
\mathcal{D}^{\gamma} f(x):=\frac{1}{\Gamma(n-\gamma)}\left(\frac{d}{d x}\right)^{n} \int_{0}^{x}(x-y)^{n-\gamma-1} f(y) d y,
$$

where $n=[\gamma]+1$, and $[\gamma]$ is the integer part of $\gamma$. 
By [10, Lemma 2.2] the Green's function associated with problem (1.1) is given by

$$
G_{\alpha}(x, y)=\frac{1}{\Gamma(\alpha)} \begin{cases}x^{\alpha-1}(1-y)^{\alpha-1}-(x-y)^{\alpha-1} & \text { for } 0 \leq y \leq x \leq 1 \\ x^{\alpha-1}(1-y)^{\alpha-1} & \text { for } 0 \leq x \leq y \leq 1\end{cases}
$$

Lemma 2.2 The Green's function $G_{\alpha}(x, y)$ has the following properties:

(i) $G_{\alpha}(x, y)$ is a nonnegative continuous function on $[0,1] \times[0,1]$.

(ii) For all $x, y \in[0,1]$, we have

$$
H_{\alpha}(x, y) \leq G_{\alpha}(x, y) \leq(\alpha-1) H_{\alpha}(x, y)
$$

where $H_{\alpha}(x, y):=\frac{1}{\Gamma(\alpha)} x^{\alpha-2}(1-y)^{\alpha-2} \min (x, y)(1-\max (x, y))$.

Proof It is obvious that (i) holds. Now we prove (ii). From (2.1), for all $x, y \in(0,1)$, we have

$$
\begin{aligned}
\Gamma(\alpha) G_{\alpha}(x, y) & =x^{\alpha-1}(1-y)^{\alpha-1}-\left((x-y)^{+}\right)^{\alpha-1} \\
& =x^{\alpha-1}(1-y)^{\alpha-1}\left(1-\left(\frac{(x-y)^{+}}{x(1-y)}\right)^{\alpha-1}\right) .
\end{aligned}
$$

Since for $\lambda>0$ and $t \in[0,1]$,

$$
\min (1, \lambda)(1-t) \leq 1-t^{\lambda} \leq \max (1, \lambda)(1-t)
$$

we deduce that

$$
1-\frac{(x-y)^{+}}{x(1-y)} \leq 1-\left(\frac{(x-y)^{+}}{x(1-y)}\right)^{\alpha-1} \leq(\alpha-1)\left(1-\frac{(x-y)^{+}}{x(1-y)}\right) .
$$

Using this fact and (2.4), we obtain

$$
x(1-y)-(x-y)^{+} \leq \frac{\Gamma(\alpha) G_{\alpha}(x, y)}{x^{\alpha-2}(1-y)^{\alpha-2}} \leq(\alpha-1)\left(x(1-y)-(x-y)^{+}\right) .
$$

Hence estimates (2.2) follow from

$$
x(1-y)-(x-y)^{+}=\min (x, y)(1-\max (x, y)) .
$$

Remark 2.3 In [1, Lemma 2.2], the authors stated that for all $x, y \in[0,1]$,

(i) $x^{\alpha-1}(1-x) y(1-y)^{\alpha-1} \leq \Gamma(\alpha) G_{\alpha}(x, y) \leq(\alpha-1) y(1-y)^{\alpha-1}$,

(ii) $x^{\alpha-1}(1-x) y(1-y)^{\alpha-1} \leq \Gamma(\alpha) G_{\alpha}(x, y) \leq(\alpha-1) x^{\alpha-1}(1-x)$.

Note that since for all $x, y \in[0,1]$,

$$
x y \leq \min (x, y) \quad \text { and } \quad(1-x)(1-y) \leq(1-\max (x, y))
$$

we get

$$
x^{\alpha-1}(1-x) y(1-y)^{\alpha-1} \leq \Gamma(\alpha) H_{\alpha}(x, y) \leq \min \left(x^{\alpha-1}(1-x), y(1-y)^{\alpha-1}\right) .
$$

Combining this fact with (2.2), we immediately obtain inequalities (i) and (ii). 
Therefore estimates (2.2) improve those stated in [1, Lemma 2.2].

Lemma 2.4 Let $q \in C((0,1),[0, \infty))$ and assume that $0<M_{q, \alpha}<\infty$. Then

$$
M_{q, \alpha+1} \leq M \leq M_{q, \alpha}
$$

where $M$ is the constant defined by (1.5).

Proof Let

$$
E=\left\{a>0: \int_{0}^{1} G_{\alpha}(x, y) h(y) q(y) d y \leq a h(x), x \in[0,1]\right\},
$$

where $h(x):=x^{\alpha-1}(1-x), x \in[0,1]$.

By (2.2) we obtain

$$
\begin{aligned}
& \int_{0}^{1} G_{\alpha}(x, y) h(y) q(y) d y \\
& \quad \leq \frac{1}{\Gamma(\alpha-1)} x^{\alpha-2} \int_{0}^{1} y^{\alpha-1}(1-y)^{\alpha-1} \min (x, y)(1-\max (x, y)) q(y) d y \\
& \quad \leq M_{q, \alpha} h(x) .
\end{aligned}
$$

It follows that $E \neq \emptyset$ and $M \leq M_{q, \alpha}$, where $M:=\inf E$.

On the other hand, using again (2.2) and that

$\min (x, y)(1-\max (x, y)) \geq x y(1-x)(1-y)$ for $x, y \in[0,1]$, we deduce that for any $a \in E$,

$$
\begin{aligned}
a h(x) & \geq \frac{1}{\Gamma(\alpha)} x^{\alpha-2} \int_{0}^{1} y^{\alpha-1}(1-y)^{\alpha-1} \min (x, y)(1-\max (x, y)) q(y) d y \\
& \geq \frac{1}{\Gamma(\alpha)} x^{\alpha-2} \int_{0}^{1} y^{\alpha-1}(1-y)^{\alpha-1} x y(1-x)(1-y) q(y) d y \\
& =h(x) M_{q, \alpha+1} .
\end{aligned}
$$

Hence for each $a \in E$,

$$
a \geq M_{q, \alpha+1}
$$

Therefore $M \geq M_{q, \alpha+1}$, that is, $M \in\left[M_{q, \alpha+1}, M_{q, \alpha}\right]$.

Remark 2.5 From Lemma 2.4 it is obvious that if $M_{q, \alpha}<1$, then

$M:=\inf E<1$. Note that the inequality $M_{q, \alpha}<1$ can be verified for a large class of functions $q$, including the singular cases. For example, let

$B(a, b):=\int_{0}^{1} t^{a-1}(1-t)^{b-1} d t$ for $a>0$ and $b>0$.

Then by using MATLAB we obtain

(i) If $q \in C((0,1))$ with $q>0$ and $\|q\|_{\infty} \leq 1$, then

$$
M_{q, \alpha} \leq \frac{B(\alpha, \alpha)}{\Gamma(\alpha-1)}<1
$$


(ii) If $q(x):=(1-x)^{-\frac{\alpha}{2}}$, then

$$
M_{q, \alpha}=\frac{B\left(\alpha, \frac{\alpha}{2}\right)}{\Gamma(\alpha-1)}<1
$$

(iii) If $q(x):=x^{-\frac{\alpha}{3}}(1-x)^{-\frac{\alpha}{2}}$, then

$$
M_{q, \alpha}=\frac{B\left(\frac{2 \alpha}{3}, \frac{\alpha}{2}\right)}{\Gamma(\alpha-1)}<1
$$

\section{Existence and uniqueness}

We need the following useful lemma.

Lemma 3.1 Let $2<\alpha<3$, and let $\varphi$ be a function such that $x \rightarrow(1-x)^{\alpha-1} \varphi(x) \in C((0,1)) \cap$ $L^{1}((0,1))$. Then the unique continuous solution of the problem

$$
\left\{\begin{array}{l}
\mathcal{D}^{\alpha} v(x)=-\varphi(x), \quad x \in(0,1), \\
v(0)=v^{\prime}(0)=v(1)=0,
\end{array}\right.
$$

is given by

$$
V \varphi(x):=\int_{0}^{1} G_{\alpha}(x, y) \varphi(y) d y .
$$

Proof Let $\varphi$ be a function such that $x \rightarrow(1-x)^{\alpha-1} \varphi(x) \in C((0,1)) \cap L^{1}((0,1))$. Since by Lemma 2.2, $G_{\alpha}(x, y)$ belongs to $C([0,1] \times[0,1])$ with

$$
0 \leq G_{\alpha}(x, y) \leq \frac{1}{\Gamma(\alpha-1)}(1-y)^{\alpha-1},
$$

we deduce by the dominated convergence theorem that $V \varphi \in C([0,1])$

and $V \varphi(0)=V \varphi(1)=0$. Therefore $I^{3-\alpha}(V|\varphi|)$ is bounded on [0,1]. By Fubini's theorem we obtain

$$
\begin{aligned}
I^{3-\alpha}(V \varphi)(x) & =\frac{1}{\Gamma(3-\alpha)} \int_{0}^{x}(x-y)^{2-\alpha} V \varphi(y) d y \\
& =\int_{0}^{1} K(x, r) \varphi(r) d r
\end{aligned}
$$

where $K(x, r):=\frac{1}{\Gamma(3-\alpha)} \int_{0}^{x}(x-y)^{2-\alpha} G_{\alpha}(y, r) d y$.

Simple calculation gives

$$
K(x, r)=\frac{1}{2} x^{2}(1-r)^{\alpha-1}-\frac{1}{2}\left((x-r)^{+}\right)^{2} .
$$

Hence, for $x \in(0,1)$, we have

$$
I^{3-\alpha}(V \varphi)(x)=\frac{x^{2}}{2} \int_{0}^{1}(1-r)^{\alpha-1} \varphi(r) d r-\frac{1}{2} \int_{0}^{x}(x-r)^{2} \varphi(r) d r .
$$


This implies that

$$
\frac{d^{3}}{d x^{3}}\left(I^{3-\alpha}(V \varphi)\right)(x)=-\varphi(x)
$$

Now, since for each $y \in(0,1)$,

$$
\lim _{x \rightarrow 0} \frac{G_{\alpha}(x, y)}{x}=0 \quad \text { and } \quad 0 \leq \frac{G_{\alpha}(x, y)}{x} \leq \frac{1}{\Gamma(\alpha-1)}(1-y)^{\alpha-1}
$$

by the dominated convergence theorem we obtain $(V \varphi)^{\prime}(0)=0$.

To prove the uniqueness, let $v, w \in C([0,1])$ be two solutions of problem (3.1) and set $\theta:=v-w$. Then $\theta \in C([0,1])$, and we have

$$
\left\{\begin{array}{l}
\mathcal{D}^{\alpha} \theta(x)=0, \\
\theta(0)=\theta^{\prime}(0)=\theta(1)=0 .
\end{array}\right.
$$

By $\left[3\right.$, Corollary 2.1] there exist $c_{1}, c_{2}, c_{3} \in \mathbb{R}$ such that

$$
\theta(x)=c_{1} x^{\alpha-1}+c_{2} x^{\alpha-2}+c_{3} x^{\alpha-3}
$$

Applying the boundary conditions, we obtain $c_{3}=c_{2}=c_{1}=0$, that is, $v=w$.

Remark 3.2 The conclusion of Lemma 3.1 remains true for $\alpha=3$.

Proof of Theorem 1.4 Assume that (A1) and (A2) hold and $M<1$, where $M$ is given by (1.5). Let us prove that problem (1.1) has a unique solution $v$ in $C_{h}([0,1])$. In addition, for any $v_{0} \in C_{h}([0,1])$, the iterative sequence $v_{k}(x):=\int_{0}^{1} G_{\alpha}(x, y) f\left(y, v_{k-1}(y)\right) d y$ converges to $v$ with respect to the $h$-norm, and we have

$$
\left\|v_{k}-v\right\|_{h} \leq \frac{M^{k}}{1-M}\left\|v_{1}-v_{0}\right\|_{h}
$$

To this end, define the operator $T$ by

$$
T v(x)=\int_{0}^{1} G_{\alpha}(x, y) f(y, v(y)) d y, x \in[0,1], v \in C_{h}([0,1]) .
$$

We claim that $T$ is a contraction operator from $\left(C_{h}([0,1]),\|\cdot\|_{h}\right)$ into itself. Let $v \in C_{h}([0,1])$, and let $\sigma>0$ be such that $|v(x)| \leq \sigma h(x)$ for all $x \in[0,1]$. Since by Lemma 2.2(ii), $0 \leq G_{\alpha}(x, y) \leq \frac{1}{\Gamma(\alpha-1)}(1-y)^{\alpha-2}$, it follows from $(A 2)$ that

$$
\begin{aligned}
\left|G_{\alpha}(x, y) f(y, v(y))\right| & \leq \frac{1}{\Gamma(\alpha-1)}(1-y)^{\alpha-2}(|f(y, v(y))-f(y, 0)|+|f(y, 0)|) \\
& \leq \frac{1}{\Gamma(\alpha-1)}(1-y)^{\alpha-2}(q(y)|v(y)|+|f(y, 0)|) \\
& \leq \frac{1}{\Gamma(\alpha-1)}\left(\sigma y^{\alpha-1}(1-y)^{\alpha-1} q(y)+(1-y)^{\alpha-2}|f(y, 0)|\right) .
\end{aligned}
$$


Since $G_{\alpha}(x, y)$ is continuous on $[0,1] \times[0,1]$, by $(\mathrm{A} 1)-(\mathrm{A} 2)$ and the dominated convergence theorem we deduce that $T v \in C([0,1])$.

Furthermore, from Lemma 2.2(ii) we have

$$
0 \leq G_{\alpha}(x, y) \leq \frac{1}{\Gamma(\alpha-1)} h(x)(1-y)^{\alpha-2}
$$

Hence by using (3.3) and similar arguments as before we obtain

$$
|T \nu(x)| \leq\left[\sigma M_{q, \alpha}+\frac{1}{\Gamma(\alpha-1)} \int_{0}^{1}(1-y)^{\alpha-2}|f(y, 0)| d y\right] h(x)
$$

and thus $T\left(C_{h}([0,1])\right) \subset C_{h}([0,1])$.

Next, for any $v, w \in C_{h}([0,1])$, by using $(A 2)$ we obtain that for $x \in[0,1]$,

$$
\begin{aligned}
|T v(x)-T w(x)| & \leq \int_{0}^{1} G_{\alpha}(x, y)|f(y, v(y))-f(y, w(y))| d y \\
& \leq \int_{0}^{1} G_{\alpha}(x, y) q(y)|v(y)-w(y)| d y \\
& \leq\|v-w\|_{h} \int_{0}^{1} G_{\alpha}(x, y) q(y) h(y) d y \\
& \leq M\|v-w\|_{h} h(x) .
\end{aligned}
$$

Hence

$$
\|T v-T w\|_{h} \leq M\|v-w\|_{h}
$$

Since $M<1, T$ becomes a contraction operator in $C_{h}([0,1])$. So there exists a unique $v \in C_{h}([0,1])$ satisfying

$$
v(x)=\int_{0}^{1} G_{\alpha}(x, y) f(y, v(y)) d y, \quad x \in(0,1) .
$$

It remains to prove that $v$ is a solution of problem (1.1). Indeed, it is clear that $x \rightarrow(1-x)^{\alpha-1} f(x, v(x)) \in C((0,1))$. Next, by using $(A 2)$ and $v \in C_{h}([0,1])$ we obtain

$$
\begin{aligned}
\left|(1-x)^{\alpha-1} f(x, v(x))\right| & \leq(1-x)^{\alpha-1}(|f(x, v(x))-f(x, 0)|+|f(x, 0)|) \\
& \leq(1-x)^{\alpha-1}(q(x)|v(x)|+|f(x, 0)|) \\
& \leq \sigma x^{\alpha-1}(1-x)^{\alpha-1} q(x)+(1-x)^{\alpha-2}|f(x, 0)| .
\end{aligned}
$$

Therefore by (A1) and (A2) it follows that $x \rightarrow(1-x)^{\alpha-1} f(x, v(x)) \in L^{1}((0,1))$. Hence from Lemma 3.1 we derive that $v$ is a solution of problem (1.1).

Finally, it is well known that for any $v_{0} \in C_{h}([0,1])$, the iterative sequence $v_{k}(x):=\int_{0}^{1} G_{\alpha}(x, y) f\left(y, v_{k-1}(y)\right) d y$ converges to $v$, and we have

$$
\left\|v_{k}-v\right\|_{h} \leq \frac{M^{k}}{1-M}\left\|v_{1}-v_{0}\right\|_{h}
$$


Example 3.3 Let $2<\alpha \leq 3$. Consider the problem

$$
\left\{\begin{array}{l}
\mathcal{D}^{\alpha} v(x)+q(x) \cos v=0, \quad x \in(0,1) \\
v(0)=v^{\prime}(0)=v(1)=0
\end{array}\right.
$$

where $q \in C((0,1))$ with $q>0$ and $\|q\|_{\infty} \leq 1$. Let $f(x, v):=q(x) \cos v$ for $(x, v) \in(0,1) \times \mathbb{R}$. We have $f \in C((0,1) \times \mathbb{R}, \mathbb{R})$ and

$$
\int_{0}^{1}(1-x)^{\alpha-2}|f(x, 0)| d x \leq\|q\|_{\infty} \int_{0}^{1}(1-x)^{\alpha-2} d x<\infty
$$

So assumption (A1) is verified.

On the other hand, since $v \rightarrow \cos v$ is a Lipschitz function, we obtain

$$
|f(x, v)-f(x, w)| \leq q(x)|v-w|, \quad x \in(0,1), v, w \in \mathbb{R} .
$$

By Lemma 2.4 and Remark 2.5(i) we have

$$
0<M \leq M_{q, \alpha} \leq \frac{\|q\|_{\infty}}{\Gamma(\alpha-1)} \int_{0}^{1} x^{\alpha-1}(1-x)^{\alpha-1} d x<1
$$

Hence by Theorem 1.4 problem (3.5) has a unique solution $v \in C_{h}([0,1])$.

Example 3.4 Let $2<\alpha \leq 3$ and consider the singular problem

$$
\left\{\begin{array}{l}
\mathcal{D}^{\alpha} v(x)+(1-x)^{-\frac{\alpha}{2}}(1+\sin v)=0, \quad x \in(0,1) \\
v(0)=v^{\prime}(0)=v(1)=0 .
\end{array}\right.
$$

In this case, we have $f(x, v)=(1-x)^{-\frac{\alpha}{2}}(1+\sin v)$ for $(x, v) \in(0,1) \times \mathbb{R}$.

So $f \in C((0,1) \times \mathbb{R}, \mathbb{R})$ and $\int_{0}^{1}(1-x)^{\alpha-2}|f(x, 0)| d x=\int_{0}^{1}(1-x)^{\frac{\alpha}{2}-2} d x<\infty$, that is, assumption (A1) is satisfied.

On the other hand, we clearly have

$$
|f(x, v)-f(x, w)| \leq q(x)|v-w|, \quad x \in(0,1), v, w \in \mathbb{R}
$$

where $q(x):=(1-x)^{-\frac{\alpha}{2}}$.

From Lemma 2.4 and Remark 2.5(ii) we deduce that

$$
0<M \leq M_{q, \alpha}=\frac{1}{\Gamma(\alpha-1)} \int_{0}^{1} x^{\alpha-1}(1-x)^{\frac{\alpha}{2}-1} d x<1
$$

Hence by Theorem 1.4 this problem has a unique solution $v \in C_{h}([0,1])$. In particular, for $\alpha=\frac{5}{2}$, the unique solution is approximated (see Fig. 1) by the iterative sequence $v_{k}(x):=$ $\int_{0}^{1} G_{\frac{5}{2}}(x, y)(1-y)^{-\frac{5}{4}}\left(1+\sin \left(v_{k-1}(y)\right)\right) d y$ with $v_{0}(x)=x^{\frac{3}{2}}(1-x), x \in[0,1]$. 


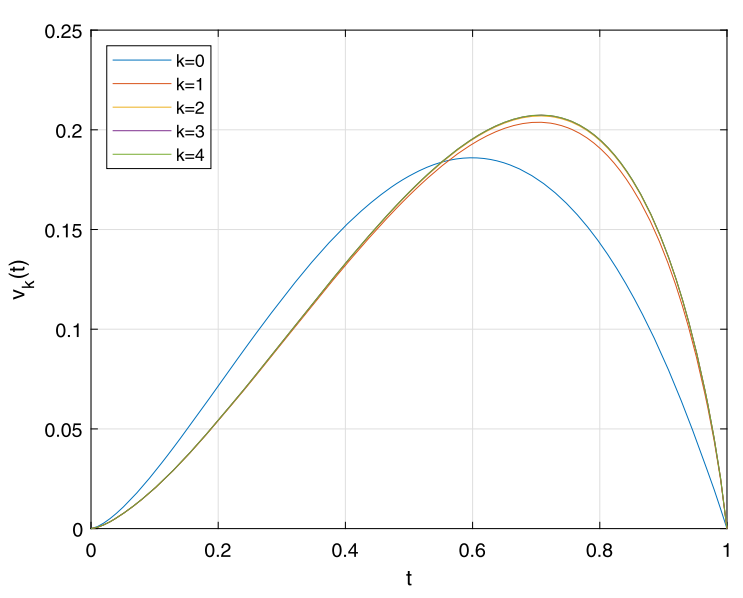

Figure 1 The approximation of the solution of problem (3.6)

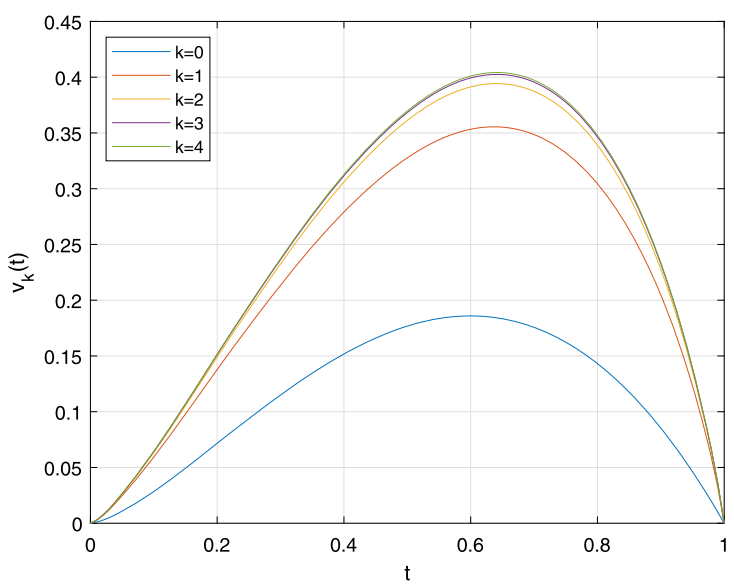

Figure 2 The approximation of the solution of problem (3.7)

Example 3.5 Consider the problem

$$
\left\{\begin{array}{l}
\mathcal{D}^{\frac{5}{2}} v(x)+x^{-\frac{5}{6}}(1-x)^{-\frac{5}{4}}(1+v)=0, \quad x \in(0,1), \\
v(0)=v^{\prime}(0)=v(1)=0 .
\end{array}\right.
$$

As in Example 3.4, we verify that assumptions (A1) and (A2) are satisfied. Therefore by Theorem 1.4 problem (3.7) has a unique solution $v \in C_{h}([0,1])$, and the iterative sequence defined by $v_{0}(x):=x^{\frac{3}{2}}(1-x), x \in[0,1]$, and

$$
v_{k}(x):=\int_{0}^{1} G_{\frac{5}{2}}(x, y) y^{-\frac{5}{6}}(1-y)^{-\frac{5}{4}}\left(1+v_{k-1}(y)\right) d y
$$

converges to $v$ (see Fig. 2). 


\section{Acknowledgements}

The authors express their thanks to the referees for their valuable suggestions and comments, which improved the presentation

\section{Funding}

The authors would like to extend their sincere appreciation to the Deanship of Scientific Research at King Saud University for its funding this Research group NO (RG-1435-043).

\section{Availability of data and materials}

Not applicable.

\section{Competing interests}

The authors declare that they have no competing interests.

\section{Authors' contributions}

All authors contributed equally to the writing of this paper. All authors read and approved the final manuscript.

\section{Author details}

${ }^{1}$ Mathematics Department, College of Science, King Saud University, P.O. Box 2455, 11451, Riyadh, Saudi Arabia. ${ }^{2}$ Department of Mathematics, College of Sciences and Arts, Rabigh Campus, King Abdulaziz University, P.O. Box 344, 21911, Rabigh, Saudi Arabia. ${ }^{3}$ Faculté des Sciences de Tunis, Université de Tunis El Manar, LR10ES09 Modélisation mathématique, analyse harmonique et théorie du potentiel, 2092, Tunis, Tunisie.

\section{Publisher's Note}

Springer Nature remains neutral with regard to jurisdictional claims in published maps and institutional affiliations.

Received: 24 October 2020 Accepted: 13 December 2020 Published online: 07 January 2021

\section{References}

1. Zou, Y., He, G.: On the uniqueness of solutions for a class of fractional differential equations. Appl. Math. Lett. 74 68-73 (2017)

2. Diethelm, K.: The Analysis of Fractional Differential Equations. Lecture Notes in Mathematics, vol. 2004. Springer, Berlin (2010)

3. Kilbas, A.A., Srivastava, H.M., Trujillo, J.J.: Theory and Applications of Fractional Differential Equations. North-Holland Mathematics Studies, vol. 204. Elsevier, Amsterdam (2006)

4. Atangana, A., Akgül, A.: Analysis of new trends of fractional differential equations. In: Fractional Order Analysis, pp. 91-111. Wiley, USA (2020)

5. Bai, Z:: On positive solutions of a nonlocal fractional boundary value problem. Nonlinear Anal. 72(2), 916-924 (2010)

6. Bai, Z., Zhang, S., Sun, S., Yin, C.: Monotone iterative method for fractional differential equations. Electron. J. Differ. Equ. 2016, $6(2016)$

7. Cui, Y.: Uniqueness of solution for boundary value problems for fractional differential equations. Appl. Math. Lett. 51 48-54 (2016)

8. Liang, S., Zhang, J.: Positive solutions for boundary value problems of nonlinear fractional differential equation. Nonlinear Anal. 71(11), 5545-5550 (2009)

9. Zhang, X., Liu, L., Wu, Y.: The uniqueness of positive solution for a fractional order model of turbulent flow in a porous medium. Appl. Math. Lett. 37, 26-33 (2014)

10. Zhang, X., Liu, L., Wu, Y: Multiple positive solutions of a singular fractional differential equation with negatively perturbed term. Math. Comput. Model. 55(3-4), 1263-1274 (2012)

\section{Submit your manuscript to a SpringerOpen ${ }^{\circ}$ journal and benefit from:}

- Convenient online submission

Rigorous peer review

- Open access: articles freely available online

- High visibility within the field

- Retaining the copyright to your article

Submit your next manuscript at $\gg$ springeropen.com 